\title{
Visualizing microscale heterogeneity of pharmaceutical samples - from solid dosages to living cells
}

\section{Dan Fu}

University of Washington, Seattle, Washington, United States

Stability and bioavailability are essential design parameters when developing pharmaceutical products. Solid dosage formulation and protein drug formulation face unique analytical challenges when measuring their stability and bioavailability. Due to the heterogeneity of active pharmaceutical ingredients (APIs) and their interaction with excipients, it is important to visualize the characterize micron-millimeter size API particles and their physical and chemical forms. Bulk spectroscopy techniques such as X-ray spectroscopy, FTIR, Raman, NMR, Mass Spectrometry, UV-Vis, and dynamic light scattering only measure the average property of the formulation. Microscopy techniques such as TEM, SEM, AFM, XRay microscopy, bright field/dark field microscopy, and fluorescence microscopy lack the chemical sensitivity to monitor subtle chemical changes that affect drug stability. We have recently demonstrated that stimulated Raman scattering microscopy (SRS), a powerful chemical imaging tool that has found increasing use in biomedical sciences, is a valuable tool for investigating the stability and bioavailability of pharmaceutical samples. SRS microscopy is a nonlinear optical imaging method that leverages the coherent excitation of Raman transitions of molecules to allow rapid three-dimensional (3D) chemical mapping of any sample with submicron spatial resolution. ${ }^{1,2}$ It combines the advantage of microscopy and spectroscopy techniques. The major applications have focused on imaging tissue structure for pathology application and cell metabolism. ${ }^{3}$ Here we will present three different applications of SRS microscopy in pharmaceutical research: 1. We use SRS microscopy to observe and quantify API size, shape, dissolution, and chemical reaction with recipients in solid dosage formulation. We show that SRS can directly monitor microscale chemical heterogeneity and chemical stability in situ over a long period of time. $^{4,5} 2$. We apply SRS microscopy to investigate the formation of protein aggregation in protein drug formulation. We demonstrate that SRS can measure particle shape, size, and chemical composition, providing critical information on the origin of aggregated particles. 3. We apply SRS to quantify tyrosine kinase inhibitor drug uptake into living cells. Small molecule drug transport across cell membrane is a key step to exert its function. We show that SRS can quantify selective accumulation of drugs in both 2D and 3D cell cultures. These three diverse applications highlight the unique power of SRS microscopy to tackle a wide range of pharmaceutical problems associated with drug stability and bioavailability.

\section{References}

1. Freudiger CW, Min W, Saar BG, Lu S, Holtom GR, He C, Tsai JC, Kang JX, Xie XS. Label-Free Biomedical Imaging with High Sensitivity by Stimulated Raman Scattering Microscopy. Science. 2008;322(5909):1857-61.

2. Fu D. Quantitative chemical imaging with stimulated Raman scattering microscopy. Curr Opin Chem Biol. 2017;39:24-31.

3. Hill AH, Fu D. Cellular Imaging Using Stimulated Raman Scattering Microscopy. Anal Chem. 2019;91(15):9333-42. 
4. Figueroa B, Nguyen T, Sotthivirat S, Xu W, Rhodes T, Lamm MS, Smith RL, John CT, Su Y, Fu D. Detecting and Quantifying Microscale Chemical Reactions in Pharmaceutical Tablets by Stimulated Raman Scattering Microscopy. Anal Chem. 2019;91(10):6894-901.

5. Francis AT, Nguyen TT, Lamm MS, Teller R, Forster SP, Xu W, Rhodes T, Smith RL, Kuiper J, Su Y, Fu D. In Situ Stimulated Raman Scattering (SRS) Microscopy Study of the Dissolution of SustainedRelease Implant Formulation. Mol Pharm. 2018;15(12):5793-801. 